\title{
Ameloblastic Carcinoma-Secondary Type (Dedifferentiated)
}

National Cancer Institute

\section{Source}

National Cancer Institute. Ameloblastic Carcinoma-Secondary Type (Dedifferentiated). NCl Thesaurus. Code C7496.

A very rare ameloblastic carcinoma that originates from a pre-existing benign ameloblastoma. 